\title{
Usage of social and behavioral change communication approaches for effective risk communication to prevent and manage COVID-19 in Nepal
}

\author{
Kiran Bam ${ }^{1}$, Rajshree Thapa ${ }^{2}$, Kiran Acharya ${ }^{3}$, Navaraj Bhattarai ${ }^{4}$, and Ashmin \\ Bhattarai $^{1}$ \\ ${ }^{1}$ Public health professional working in Nepal \\ ${ }^{2}$ Monash University \\ ${ }^{3}$ New ERA \\ ${ }^{4}$ Nepal Public Health Research and Development Centre
}

August 11, 2020

\begin{abstract}
Coronavirus disease 2019 (COVID-19) has appeared as the biggest challenge of present time as our health systems are struggling to contain the spread of the virus. As COVID-19 developed rapidly into a pandemic, people need to acquire and apply health information, and adapt into their behavior right away. However, there exists a global epidemic of misinformation alongside this pandemic. People at risk need to be able to take informed decisions on mitigating the effects of the disease outbreak and practice protective and preventive actions. This affirms the urgency and importance of forming a dedicated risk communication team in the country to design and deliver risk communication packages with the SBCC approach addressing the need of subpopulations dwelling at all levels.
\end{abstract}

\section{Main text}

Low -and middle-income countries face an additional challenge in responding to the surging cases of COVID19 globally. These countries are already competing with the limited resources and triple burden of diseases. The cases are soaring everyday as the testing services are gradually expanded. The current pandemic have high rates of infection, notable morbidity and mortality, inadequate protective or therapeutic measures, and substantial increases in cases or case-fatality rates ${ }^{[1]}$. Most significant of these factors could be the perception, communication and management of the risk presented by COVID-19. It is vital to locate the analysis with respect to the risk communication ${ }^{[1]}$. While few countries have already reported successful containment of the cases, the situation in South Asia is reverse with initial low rate of infection and a constant rise in cases with expansion of testing services.

In most South Asian countries, the nationwide lock-down has been gradually eased, not because the cases of COVID-19 have declined but out of the compulsion and pressure from the socio-economic sectors. As COVID-19 developed rapidly into a pandemic, people needed to acquire and apply health information, and adapt into their behavior right away ${ }^{[2]}$. Health information offering simple and practical solutions that include hand hygiene, physical distancing and use of protective measures are widely available. There are global initiatives that played a pivotal role in gradually controlling the virus in many countries. These initiatives aimed at acting as myth busters to enable fact-checking and providing credible sources relating to COVID-19 ${ }^{[3]}$. However, there exists a global epidemic of misinformation alongside this pandemic ${ }^{[4]}$. As no country is immune to misinformation, people should be enabled to take informed decisions on mitigating the 
effects of a disease outbreak and practice protective and preventive actions ${ }^{[5]}$. This affirms the urgency and importance of risk communication ${ }^{[6]}$. Risk communication refers to the real-time exchange of information, advice and opinions between health experts or officials and general people who face a threat to their survival, health or socio-economic well-being ${ }^{[5]}$. Global evidence shows that effective risk communication are helpful to avert new infections, management of the existing infections, deal with the "Infodemics" and engage communities in pandemic response ${ }^{[7-9]}$. In such pandemic, where the situations tend to change promptly with rapid flow of information, the role of risk communication is ever so important. Therefore, along with the transmission of reliable and updated information, risk communication should also appropriately enthuse change in behavior ${ }^{[10]}$. The authors propose adoption of risk communication framework emphasizing on 4Es, namely: Evidence, Execute, Empower and Engage as depicted in Figure 1.

Nationwide lockdown, physical distancing measures, different cues to action have been brought into place across Nepal to reduce transmission of SARS-Cov-2. Despite these efforts supplemented with expansion of testing facilities across the nation, the number of infections is increasing every day. Though the messages are simple, they are not inevitably modest to implement for all. Even if everybody had reliable and consistent information, behavior change would still be a challenge. This will require communication to develop selfawareness that is carelessly habitual to adjust to the new normal situation ${ }^{[11]}$. Several aspects of social and cultural contexts affect the level and hustle of behavior change. Therefore, there require significant shift in behavior during the pandemic while the immense flow of health information and perspectives on the pandemic is unparalleled and speckled ${ }^{[11]}$. There has been debate in the role of the mass media in risk communication within health and there should be one door system of information flow. The media coverage during the outbreak of SARS in the beginning of 21st century was excessive, sometimes inaccurate, and sensationalist ${ }^{[12]}$. Social media platforms albeit, a widely available platform to disseminate health information, these platforms are also the source of misinformation.

As of August 10, Nepal reported 23,310 positive cases (with 16,493 cases recovered) and 79 deaths, with the majority of the cases returning from abroad ${ }^{[13]}$. The Government of Nepal (GoN) in collaboration with non-government counterparts is developing and disseminating messages on prevention, testing, treatment and care of COVID-19. Ministry of Health and Population (MoHP) has been coordinating for messaging at designated stations of the air or ground routes, responding with the frequently asked questions from the public, and developing and disseminating information, education and communication (IEC) materials. MoHP has also developed guidelines and mobilized contact-tracing teams. Likewise, National Health Education Information and Communication Centre (NHEICC) under the MoHP is the focal point for social and behavioral change communication (SBCC) related activities. Religious leaders are oriented to sensitize wide range of stakeholders. The World Health Organization has been instrumental in developing and promoting SBCC materials and sharing with concerned authorities for wider circulation in both Nepali and English languages via websites. COVID-19 related IEC, and training materials distributed in key locations via stakeholders. Collaborative work with partner agencies and mobile networks for pushing the COVID-19 awareness messages in interactive voice response and short message service formats is being undertaken along with messaging through mass media.

Further, MoHP organizes daily press releases and prepares daily situation reports disseminated via various mass media, social media platforms and websites aided with sign language targeting people with hearing disorder. Some of the provincial ministries have also initiated such practice. There are designated hotline numbers and mobile numbers to provide information on COVID-19. However, consistency of the messages delivered through these various platforms is still an issue. Some other measures include orientation to media on COVID-19, crash course on risk communication to MOHP officials, social media: COVID-19 web portal,Hamro Swasthya mobile application, MoHP official Facebook page and Viber community.

Despite these various initiatives, people are disregarding the proven cost-effective prevention strategies such as the use of masks or physical distancing measures. In crowded cities of Nepal, maintaining social distance is quite a challenge. (Figure 2: People disregarding the use of mask and physical distancing while crowding to buy fruits from a mobile vendor in Bhaktapur municipality of Nepal) Not 
only the population density but also social structures often create a hindrance in physical distancing. Public transportation being the main mode of transport, people are compelled to travel in crowded vehicles. Behavior change intervention should thus address such pragmatic challenges and devise ways to work with interdisciplinary ministries such as transport, labor, media, and environment in facilitating the change in work culture while promoting health behavior. SBCC is a gradual and continuous process. There is a need of balance between fear/risk along with benefits of such an approach. SBCC entails interactive and easy to comprehend messages along with edutainment messages. To be effective, SBCC materials and messaging need to incorporate ideas, images, and logic that will promote comprehension among lay public. Small simple practical nudges such as the use of metal bars in front of local shops can help maintain physical distancing. Strict regulation on mask use, availability sanitizers or hand washing facilities at the shopping malls, stores, food outlets, public vehicles, social gatherings until they gradually develop as our habitual practice.

Other issue is the control of rumors, fear and stigma associated with the disease. Wide spread of fake news and rumors from various social media platforms creates unnecessary fear and stigmatization of infected with COVID-19. There are challenges around handling media and journalists and selecting channels of dissemination, although media monitoring is being undertaken and addressed during experts' sharing. SBCC requires information sharing and establishing networks of working relationships among individuals, groups similar to the socio-ecological model. This is time to deal with epidemics rather than infodemics through mobilization of the technical experts and media influencers and strict media monitoring.

It is crucial to recognize the needs of specific groups such as children and adults with disabilities, people who are illiterate while engaging with larger populations within a community in the response to COVID-19. They might experience barriers to retrieving information, care and support or are at higher risk of exposure and secondary impacts. This population make up an estimated nearly one sixth of the population and are often unseen and excluded. The needs of the vulnerable population should be central while designing such an intervention. Nepal's population is varied with diverse characteristics along with large topographical differences. There need to be tailored messages that enhance the public's perception with a clear "menu of options". Health Sector Emergency Response Plan developed by GoN calls NHEICC for the development of standardized messages that is to be delivered through coordinated channels and with consideration of the local needs. While the government is struggling to mitigate the effects of this global pandemic, an urgent need exists on forming a dedicated risk communication team in the country to design and deliver risk communication packages with the SBCC approach addressing the need of sub-populations dwelling at all levels. These communication interventions must display accountability by keeping the public updated on the situation, on actions carried out, and the impact of those actions in containing the spread of disease.

\section{Reference}

1. Smith RD. Responding to global infectious disease outbreaks: Lessons from SARS on the role of risk perception, communication and management.Soc Sci Med . 2006;63(12):3113-3123. doi:10.1016/j.socscimed.2006.08.004

2. Paakkari L, Okan O. COVID-19: health literacy is an underestimated problem. Lancet Public Health . 2020;5(5):e249-e250. doi:10.1016/S2468-2667(20)30086-4

3. The Covid-19 'infodemic': a new front for information professionals - Naeem - Health Information \&amp; Libraries Journal - Wiley Online Library. Accessed July 2, 2020. https://onlinelibrary.wiley.com/doi/full/10.1111/hir.12311

4. Zarocostas J. How to fight an infodemic. Lancet . 2020;395(10225):676. doi:10.1016/S01406736(20)30461-X

5. World Health Organization. Managing Epidemics Key Facts about Major Deadly Diseases .; 2018. //oemmndcbldboiebfnladdacbdfmadadm/https://www.who.int/emergencies/diseases/managingepidemics-interactive.pdf

6. Infodemic and risk communication in the era of CoV-19 Vaezi A, Javanmard SH - 
Adv Biomed Res. Accessed July 2, 2020. http://www.advbiores.net/article.asp?issn=22779175 ; year $=2020 ;$ volume $=9 ;$ issue $=1$; spage $=10 ;$ epage $=10 ;$ aulast $=$ Vaezi

7. A Voice from the frontline: the role of risk communication in managing the COVID-19 Infodemic and engaging communities in pandemic response. Journal of Communication in Healthcare . 2020;13(1):6-9. doi:10.1080/17538068.2020.1758427

8. Dickmann P, Abraham T, Sarkar S, et al. Risk communication as a core public health competence in infectious disease management: Development of the ECDC training curriculum and programme. Euro Surveill . 2016;21(14). doi:10.2807/1560-7917.ES.2016.21.14.30188

9. Vaezi A, Javanmard SH. Infodemic and risk communication in the era of CoV-19. Advanced Biomedical Research . 2020;9(1):10. doi:10.4103/abr.abr_47_20

10. Abrams EM, Greenhawt M. Risk Communication During COVID-19. J Allergy Clin Immunol Pract . 2020;8(6):1791-1794. doi:10.1016/j.jaip.2020.04.012

11. Finset A, Bosworth H, Butow P, et al. Effective health communication - a key factor in fighting the COVID-19 pandemic. Patient Educ Couns . 2020;103(5):873-876. doi:10.1016/j.pec.2020.03.027

12. Rezza G, Marino R, Farchi F, Taranto M, Superiore di Sanità I. SARS Epidemic in the Press. Emerg Infect Dis . 2004;10(2):381-382. doi:10.3201/eid1002.030743

13. Ministry of Health and Population, Ministry of Health and Population. SitRep . Accessed August 11, 2020. https://drive.google.com/drive/folders/1QhLMbT76t6Zu1sFy5qlB5aoDbHVAcnHx

\section{List of abbreviations}

SBCC Social and behavioral change communication

GoN Government of Nepal

MoHP Ministry of Health and Population

IEC Information, education and communication

NHEICC National Health Education Information and Communication Centre

\section{Declarations}

Ethics approval and consent to participate

Not applicable

Consent for publication

Not applicable

Availability of data and materials

None

Competing interests

The authors declare that they have no competing interests for this work.

Funding

None

Authors' contributions 
AHB and KB conceived the idea of the commentary, and coordinated to draft the manuscript. RT, KA and NB contributed in critically reviewing and editing the manuscript. All authors read and approved the final copy of the manuscript.

Acknowledgements

None

\section{Hosted file}

Figure 1.pptx available at https://authorea.com/users/350153/articles/475069-usage-ofsocial-and-behavioral-change-communication-approaches-for-effective-risk-communicationto-prevent-and-manage-covid-19-in-nepal

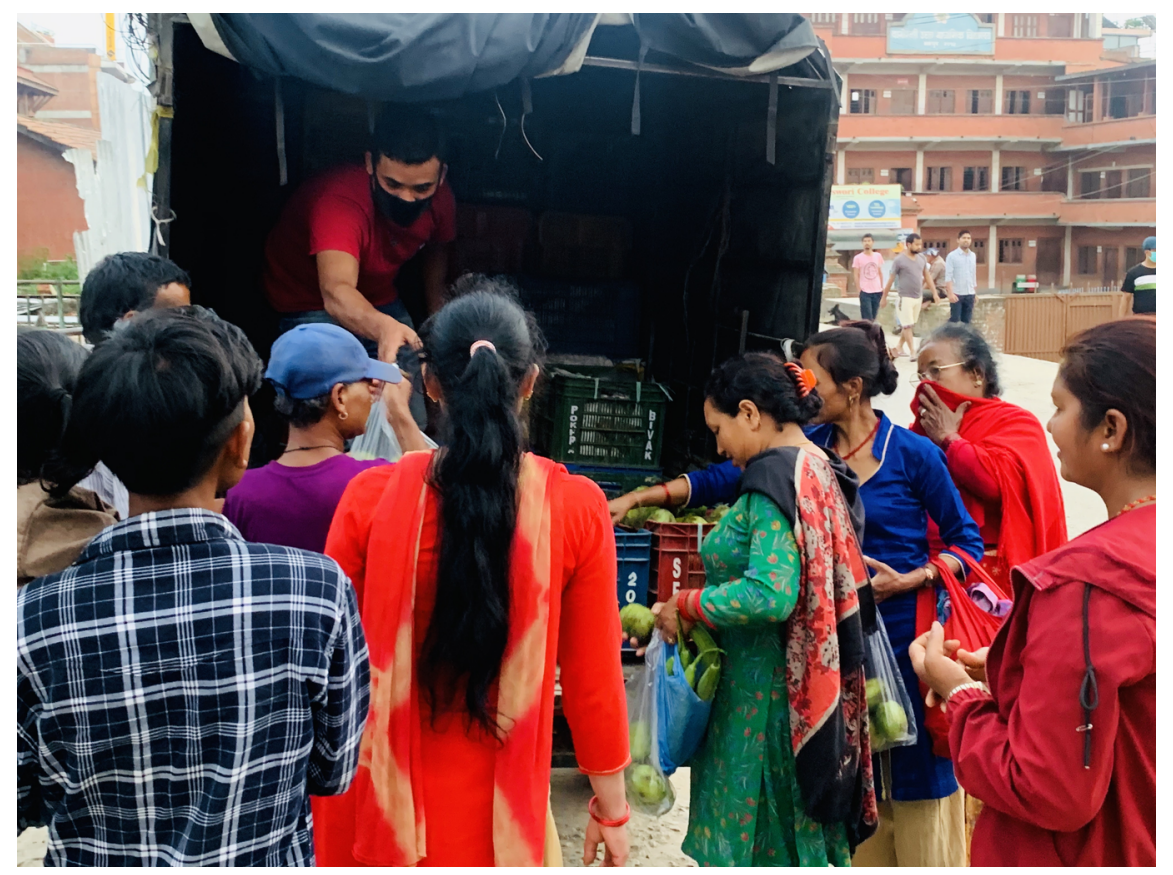

